\title{
Local Fractional Variational Iteration and Decomposition Methods for Wave Equation on Cantor Sets within Local Fractional Operators
}

\author{
Dumitru Baleanu, ${ }^{1,2,3}$ J. A. Tenreiro Machado, ${ }^{4}$ Carlo Cattani, ${ }^{5}$ \\ Mihaela Cristina Baleanu, ${ }^{6}$ and Xiao-Jun Yang ${ }^{7}$ \\ ${ }^{1}$ Department of Chemical and Materials Engineering, Faculty of Engineering, King Abdulaziz University, \\ P.O. Box 80204, Jeddah 21589, Saudi Arabia \\ ${ }^{2}$ Department of Mathematics and Computer Sciences, Faculty of Arts and Sciences, Cankaya University, \\ 06530 Ankara, Turkey \\ ${ }^{3}$ Institute of Space Sciences, Magurele, Bucharest, Romania \\ ${ }^{4}$ Department of Electrical Engineering, Institute of Engineering, Polytechnic of Porto, Rua Dr. Antonio \\ Bernardino de Almeida 431, 4200-072 Porto, Portugal \\ ${ }^{5}$ Department of Mathematics, University of Salerno, Via Ponte don Melillo, Fisciano, 84084 Salerno, Italy \\ ${ }^{6}$ Mihail Sadoveanu Theoretical High School, District 2, Street Popa Lazar No. 8, 021586 Bucharest, Romania \\ ${ }^{7}$ Department of Mathematics and Mechanics, China University of Mining and Technology, Xuzhou Campus, \\ Xuzhou, Jiangsu 221008, China \\ Correspondence should be addressed to Xiao-Jun Yang; dyangxiaojun@163.com
}

Received 9 November 2013; Accepted 8 December 2013; Published 2 January 2014

Academic Editor: Ming Li

Copyright ( 2014 Dumitru Baleanu et al. This is an open access article distributed under the Creative Commons Attribution License, which permits unrestricted use, distribution, and reproduction in any medium, provided the original work is properly cited.

We perform a comparison between the fractional iteration and decomposition methods applied to the wave equation on Cantor set. The operators are taken in the local sense. The results illustrate the significant features of the two methods which are both very effective and straightforward for solving the differential equations with local fractional derivative.

\section{Introduction}

Many problems of physics and engineering are expressed by ordinary and partial differential equations, which are termed boundary value problems. We can mention, for example, the wave, the Laplace, the Klein-Gordon, the Schrodinger's, the telegraph, the Advection, the Burgers, the KdV, the Boussinesq, and the Fisher equations and others [1].

Recently, the fractional calculus theory was recognized to be a good tool for modeling complex problems demonstrating its applicability in numerical scientific disciplines. Boundary value problems for the fractional differential equations have been the focus of several studies due to their frequent appearance in various areas, such as fractional diffusion and wave [2], fractional telegraph [3], fractional KdV [4], fractional Schrödinger [5], fractional evolution [6], fractional Navier-Stokes [7], fractional Heisenberg [8], fractional KleinGordon [9], and fractional Fisher equations [10].

Several analytical and numerical techniques were successfully applied to deal with differential equations, fractional differential equations, and local fractional differential equations (see, e.g., [1-36] and the references therein). The techniques include the heat-balance integral [11], the fractional Fourier [12], the fractional Laplace transform [12], the harmonic wavelet $[13,14]$, the local fractional Fourier and Laplace transform [15], local fractional variational iteration $[16,17]$, the local fractional decomposition [18], and the generalized local fractional Fourier transform [19] methods. 
Recently, the wave equation on Cantor sets (local fractional wave equation) was given by [35]

$$
\frac{\partial^{2 \alpha} u(x, t)}{\partial t^{2 \alpha}}-a^{2 \alpha} \frac{\partial^{2 \alpha} u(x, t)}{\partial x^{2 \alpha}}=0
$$

where the operators are local fractional ones [16-19, 35, 36].

Following (1), a wave equation on Cantor sets was proposed as follows [36]:

$$
\frac{\partial^{2 \alpha} u(x, t)}{\partial t^{2 \alpha}}-\frac{x^{2 \alpha}}{\Gamma(1+2 \alpha)} \frac{\partial^{2 \alpha} u(x, t)}{\partial x^{2 \alpha}}=0,
$$

where $u(x, t)$ is a fractal wave function.

In this paper, our purpose is to compare the local fractional variational iteration and decomposition methods for solving the local fractional differential equations. For illustrating the concepts we adopt one example for solving the wave equation on Cantor sets with local fractional operator.

Bearing these ideas in mind, the paper is organized as follows. In Section 2, we present basic definitions and provide some properties of local fractional derivative and integration. In Section 3, we introduce the local fractional variational iteration and the decomposition methods. In Section 4, we discuss one application. Finally, in Section 5 we outline the main conclusions.

\section{Mathematical Tools}

We recall in this section the notations and some properties of the local fractional operators $[15-19,35,36]$.

Definition 1 (see $[15-19,35,36])$. The function $f(x)$ is local fractional continuous, if it is valid for

$$
\left|f(x)-f\left(x_{0}\right)\right|<\varepsilon^{\alpha},
$$

where $\left|x-x_{0}\right|<\delta$, for $\varepsilon>0$ and $\varepsilon \in R$.

We notice that there are existence conditions of local fractional continuities that operating functions are righthand and left-hand local fractional continuity. Meanwhile, the right-hand local fractional continuity is equal to its lefthand local fractional continuity. For more details, see [35].

Following (4), we have [15-19, 35, 36]

$$
\rho^{\alpha}\left|x-x_{0}\right|^{\alpha} \leq\left|f(x)-f\left(x_{0}\right)\right| \leq \kappa^{\alpha}\left|x-x_{0}\right|^{\alpha}
$$

with $\left|x-x_{0}\right|<\delta$, for $\varepsilon, \delta>0$ and $\varepsilon, \delta, \kappa, \rho \in R$.

For a fractal set $F$, there is a fractal measure [35]

$$
H^{\alpha}\left(F \cap\left(x, x_{0}\right)\right)=\left(x-x_{0}\right)^{\alpha},
$$

where $f(x)$ presents a bi-Lipschitz mapping with fractal dimension $\alpha$ and $H^{\alpha}$ denotes a Hausdorff dimension.

We verify that there is a measure

$$
H^{1}\left(F \cap\left(x, x_{0}\right)\right)=x-x_{0}
$$

in the case of $\alpha=1$ and $f(x)$ is a Lipschitz mapping. If $F$ is a Cantor set, we have $H^{\ln 2 / \ln 3}\left(F \cap\left(x, x_{0}\right)\right)=\left(x-x_{0}\right)^{\ln 2 / \ln 3}$ with $\alpha=\ln 2 / \ln 3$.
Definition 2 (see $[15-19,35,36])$. The local fractional derivative of $f(x)$ at $x=x_{0}$ is defined as [16-20]

$$
f^{(\alpha)}\left(x_{0}\right)=\left.\frac{d^{\alpha} f(x)}{d x^{\alpha}}\right|_{x=x_{0}}=\lim _{x \rightarrow x_{0}} \frac{\Delta^{\alpha}\left(f(x)-f\left(x_{0}\right)\right)}{\left(x-x_{0}\right)^{\alpha}},
$$

where

$$
\Delta^{\alpha}\left(f(x)-f\left(x_{0}\right)\right) \cong \Gamma(1+\alpha) \Delta\left(f(x)-f\left(x_{0}\right)\right) .
$$

We find that the existence condition for local fractional derivative of $f(x)$ is that the right-hand local fractional derivative is equal to the left-hand local fractional derivative (see, e.g., $[16,35]$ and the references therein).

Definition 3 (see $[15-19,35,36])$. A partition of the interval $[a, b]$ is denoted as $\left(t_{j}, t_{j+1}\right), j=0, \ldots, N-1, t_{0}=a$, and $t_{N}=b$ with $\Delta t_{j}=t_{j+1}-t_{j}$ and $\Delta t=\max \left\{\Delta t_{0}, \Delta t_{1}, \Delta t_{j}, \ldots\right\}$. Local fractional integral of $f(x)$ in the interval $[a, b]$ is given by

$$
\begin{aligned}
a_{a}^{I_{b}(\alpha)} f(x) & =\frac{1}{\Gamma(1+\alpha)} \int_{a}^{b} f(t)(d t)^{\alpha} \\
& =\frac{1}{\Gamma(1+\alpha)} \lim _{\Delta t \rightarrow 0} \sum_{j=0}^{j=N-1} f\left(t_{j}\right)\left(\Delta t_{j}\right)^{\alpha} .
\end{aligned}
$$

If the functions are local fractional continuous then the local fractional derivatives and integrals exist. That is to say, operating functions have nondifferentiable and fractal properties (see [35] and the references therein).

Some properties of local fractional derivative and integrals are given in [35].

\section{Analytical Methods}

In order to illustrate two analytical methods, we investigate the nonlinear local fractional equation as follows:

$$
L_{\alpha}^{(n)} u+R_{\alpha} u=0
$$

where $L_{\alpha}^{(n)}$ is linear local fractional operators, respectively, with $n=1,2$ and $R_{\alpha}$ is linear local fractional operators of order less than $L_{\alpha}^{(n)}$.

3.1. Local Fractional Variational Iteration Method. The local fractional variational iteration algorithm is given by $[16,17]$ on the line of the formalism suggested in [35]

$$
\begin{aligned}
u_{n+1}(t)= & u_{n}(t)+\frac{1}{\Gamma(1+\alpha)} \\
& \times \int_{0}^{t} \frac{\lambda^{\alpha}}{\Gamma(1+\alpha)}\left\{L_{\alpha}^{(n)} u_{n}(s)+R_{\alpha} u_{n}(s)\right\}(d s)^{\alpha} .
\end{aligned}
$$

Here, we can construct a correction functional as follows [16, 17]:

$$
\begin{aligned}
u_{n+1}(t)= & u_{n}(t)+\frac{1}{\Gamma(1+\alpha)} \\
& \times \int_{0}^{t} \frac{\lambda^{\alpha}}{\Gamma(1+\alpha)}\left\{L_{\alpha}^{(n)} u_{n}(s)+R_{\alpha} \tilde{u}_{n}(s)\right\}(d s)^{\alpha},
\end{aligned}
$$


where $\tilde{u}_{n}$ is considered as a restricted local fractional variation; that is, $\delta^{\alpha} \widetilde{u}_{n}=0$ (for more details, see [35]).

For $n=2$, we have

$$
\lambda^{\alpha}=\frac{(s-t)^{\alpha}}{\Gamma(1+\alpha)},
$$

so that iteration is expressed as

$$
\begin{aligned}
u_{n+1}(t)= & u_{n}(t)+\frac{1}{\Gamma(1+\alpha)} \\
& \times \int_{0}^{t} \frac{(s-t)^{\alpha}}{\Gamma(1+\alpha)}\left\{L_{\alpha}^{(n)} u_{n}(s)+R_{\alpha} u_{n}(s)\right\}(d s)^{\alpha} .
\end{aligned}
$$

Finally, the solution is

$$
u(x)=\lim _{n \rightarrow \infty} u_{n}(x)
$$

3.2. Local Fractional Decomposition Method. When $L_{\alpha}^{(n)}$ in (10) is a local fractional differential operator of order $2 \alpha$, we denote it as

$$
\begin{gathered}
L_{\alpha}^{(n)}=L_{x x}^{(2 \alpha)}=\frac{\partial^{2 \alpha}}{\partial x^{2 \alpha}}, \\
R_{\alpha} u(t)=\frac{\partial^{\alpha}}{\partial x^{\alpha}} u(t)+f(t) .
\end{gathered}
$$

By defining the $n$-fold local fractional integral operator

$$
L_{\alpha}^{(-2)} m(s)={ }_{0} I_{x}{ }^{(\alpha)}{ }_{0} I_{x}{ }^{(\alpha)} m(s)
$$

we get

$$
L_{\alpha}^{(-2)} L_{\alpha}^{(2)} u(s)=L_{\alpha}^{(-2)} R_{\alpha} u(s)
$$

Thus,

$$
u(s)=r(x)+L_{\alpha}^{(-2)} R_{\alpha} u(s),
$$

where the term $r(x)$ is to be determined from the fractal initial conditions.

Therefore, we get the iterative formula as follows:

$$
u(x)=u_{0}(x)+L_{\alpha}^{(-2)} R_{\alpha} u(s),
$$

with $u_{0}(x)=r(x)$.

Hence, for $n \geq 0$, we have the following recurrence relationship:

$$
\begin{gathered}
u_{n+1}(x)=L_{\alpha}^{(-2)} R_{\alpha} u_{n}(s), \\
u_{0}(x)=r(x) .
\end{gathered}
$$

Finally, the solution can be constructed as

$$
u(x)=\lim _{n \rightarrow \infty} \phi_{n}(x)=\lim _{n \rightarrow \infty} \sum_{n=0}^{\infty} u_{n}(x) .
$$

For more details, see [18].

\section{An Illustrative Example}

In this section one example for wave equation is presented in order to demonstrate the simplicity and the efficiency of the above methods.

In (2), we consider the following initial and boundary conditions:

$$
\frac{\partial^{\alpha} u(x, 0)}{\partial t^{\alpha}}=0, \quad u(x, 0)=\frac{x^{2 \alpha}}{\Gamma(1+2 \alpha)} .
$$

Using (14) we have the iterative formula

$$
\begin{aligned}
u_{n+1}(x, t) & \\
= & u_{n}(x, t)+\frac{1}{\Gamma(1+\alpha)} \int_{0}^{t} \frac{(s-t)^{\alpha}}{\Gamma(1+\alpha)} \frac{\partial^{2 \alpha} u_{n}(x, s)}{\partial s^{2 \alpha}}(d s)^{\alpha} \\
& -\frac{1}{\Gamma(1+\alpha)} \int_{0}^{t} \frac{(s-t)^{\alpha}}{\Gamma(1+\alpha)} \frac{x^{2 \alpha}}{\Gamma(1+2 \alpha)} \frac{\partial^{2 \alpha} u_{n}(x, s)}{\partial x^{2 \alpha}}(d s)^{\alpha},
\end{aligned}
$$

where the initial value is given by

$$
u_{0}(x, t)=\frac{x^{2 \alpha}}{\Gamma(1+2 \alpha)}
$$

Thus, after computing (23) we obtain

$$
\begin{aligned}
& u_{1}(x, t) \\
& =u_{0}(x, t)+\frac{1}{\Gamma(1+\alpha)} \int_{0}^{t} \frac{(s-t)^{\alpha}}{\Gamma(1+\alpha)} \frac{\partial^{2 \alpha} u_{0}(x, s)}{\partial s^{2 \alpha}}(d s)^{\alpha} \\
& -\frac{1}{\Gamma(1+\alpha)} \int_{0}^{t} \frac{(s-t)^{\alpha}}{\Gamma(1+\alpha)} \frac{x^{2 \alpha}}{\Gamma(1+2 \alpha)} \frac{\partial^{2 \alpha} u_{0}(x, s)}{\partial x^{2 \alpha}}(d s)^{\alpha} \\
& =u_{0}(x, t)+\frac{1}{\Gamma(1+\alpha)} \int_{0}^{t} \frac{(s-t)^{\alpha}}{\Gamma(1+\alpha)}\left\{-\frac{x^{2 \alpha}}{\Gamma(1+2 \alpha)}\right\}(d s)^{\alpha} \\
& =\frac{x^{2 \alpha}}{\Gamma(1+2 \alpha)}\left(1+\frac{t^{2 \alpha}}{\Gamma(1+2 \alpha)}\right) \text {, } \\
& u_{2}(x, t) \\
& =u_{1}(x, t)+\frac{1}{\Gamma(1+\alpha)} \int_{0}^{t} \frac{(s-t)^{\alpha}}{\Gamma(1+\alpha)} \frac{\partial^{2 \alpha} u_{1}(x, s)}{\partial s^{2 \alpha}}(d s)^{\alpha} \\
& -\frac{1}{\Gamma(1+\alpha)} \int_{0}^{t} \frac{(s-t)^{\alpha}}{\Gamma(1+\alpha)} \frac{x^{2 \alpha}}{\Gamma(1+2 \alpha)} \frac{\partial^{2 \alpha} u_{1}(x, s)}{\partial x^{2 \alpha}}(d s)^{\alpha} \\
& =u_{1}(x, t)+\frac{1}{\Gamma(1+\alpha)} \\
& \times \int_{0}^{t} \frac{(s-t)^{\alpha}}{\Gamma(1+\alpha)}\left\{-\frac{x^{2 \alpha}}{\Gamma(1+2 \alpha)} \frac{s^{2 \alpha}}{\Gamma(1+2 \alpha)}\right\}(d s)^{\alpha} \\
& =\frac{x^{2 \alpha}}{\Gamma(1+2 \alpha)}\left(1+\frac{t^{2 \alpha}}{\Gamma(1+2 \alpha)}+\frac{t^{4 \alpha}}{\Gamma(1+4 \alpha)}\right) \text {, }
\end{aligned}
$$




$$
\begin{aligned}
& u_{3}(x, t) \\
& =u_{2}(x, t)+\frac{1}{\Gamma(1+\alpha)} \int_{0}^{t} \frac{(s-t)^{\alpha}}{\Gamma(1+\alpha)} \frac{\partial^{2 \alpha} u_{2}(x, s)}{\partial s^{2 \alpha}}(d s)^{\alpha} \\
& -\frac{1}{\Gamma(1+\alpha)} \int_{0}^{t} \frac{(s-t)^{\alpha}}{\Gamma(1+\alpha)} \frac{x^{2 \alpha}}{\Gamma(1+2 \alpha)} \frac{\partial^{2 \alpha} u_{2}(x, s)}{\partial x^{2 \alpha}}(d s)^{\alpha} \\
& =u_{2}(x, t)+\frac{1}{\Gamma(1+\alpha)} \\
& \times \int_{0}^{t} \frac{(s-t)^{\alpha}}{\Gamma(1+\alpha)}\left\{-\frac{x^{2 \alpha}}{\Gamma(1+2 \alpha)} \frac{t^{4 \alpha}}{\Gamma(1+4 \alpha)}\right\}(d s)^{\alpha} \\
& =\frac{x^{2 \alpha}}{\Gamma(1+2 \alpha)} \sum_{i=0}^{3} \frac{t^{2 i \alpha}}{\Gamma(1+2 i \alpha)} \text {, } \\
& u_{4}(x, t) \\
& =u_{3}(x, t)+\frac{1}{\Gamma(1+\alpha)} \int_{0}^{t} \frac{(s-t)^{\alpha}}{\Gamma(1+\alpha)} \frac{\partial^{2 \alpha} u_{3}(x, s)}{\partial s^{2 \alpha}}(d s)^{\alpha} \\
& -\frac{1}{\Gamma(1+\alpha)} \int_{0}^{t} \frac{x^{2 \alpha}}{\Gamma(1+2 \alpha)} \frac{\partial^{2 \alpha} u_{3}(x, s)}{\partial x^{2 \alpha}}(d s)^{\alpha} \\
& =u_{3}(x, t)+\frac{1}{\Gamma(1+\alpha)} \\
& \times \int_{0}^{t} \frac{(s-t)^{\alpha}}{\Gamma(1+\alpha)}\left\{-\frac{x^{2 \alpha}}{\Gamma(1+2 \alpha)} \frac{t^{6 \alpha}}{\Gamma(1+6 \alpha)}\right\}(d s)^{\alpha} \\
& =\frac{x^{2 \alpha}}{\Gamma(1+2 \alpha)} \sum_{i=0}^{4} \frac{t^{2 i \alpha}}{\Gamma(1+2 i \alpha)}, \\
& u_{n}(x, t) \\
& =u_{n-1}(x, t)+\frac{1}{\Gamma(1+\alpha)} \int_{0}^{t} \frac{(s-t)^{\alpha}}{\Gamma(1+\alpha)} \frac{\partial^{2 \alpha} u_{n-1}(x, s)}{\partial s^{2 \alpha}}(d s)^{\alpha} \\
& -\frac{1}{\Gamma(1+\alpha)} \\
& \times \int_{0}^{t} \frac{(s-t)^{\alpha}}{\Gamma(1+\alpha)} \frac{x^{2 \alpha}}{\Gamma(1+2 \alpha)} \frac{\partial^{2 \alpha} u_{n-1}(x, s)}{\partial x^{2 \alpha}}(d s)^{\alpha} \\
& =\frac{x^{2 \alpha}}{\Gamma(1+2 \alpha)} \sum_{i=0}^{n} \frac{t^{2 i \alpha}}{\Gamma(1+2 i \alpha)} \text {. }
\end{aligned}
$$

Hence, from (27) we obtain the solution of (3) as

$$
u(x)=\lim _{n \rightarrow \infty} u_{n}(x)=\frac{x^{2 \alpha}}{\Gamma(1+2 \alpha)} \cosh _{\alpha}\left(t^{\alpha}\right) .
$$

Here, from (21) we get

$$
\begin{gathered}
u_{n+1}(x, t)={ }_{0} I_{t}{ }^{(\alpha)}{ }_{0} I_{t}{ }^{(\alpha)} \frac{x^{2 \alpha}}{\Gamma(1+2 \alpha)} \frac{\partial^{2 \alpha} u_{n}(x, s)}{\partial x^{2 \alpha}}, \\
u_{0}(x, t)=\frac{x^{2 \alpha}}{\Gamma(1+2 \alpha)} .
\end{gathered}
$$

Therefore, from (29) we give the components as follows:

$$
\begin{gathered}
u_{0}(x, t)=\frac{x^{2 \alpha}}{\Gamma(1+2 \alpha)}, \\
u_{1}(x, t)=\frac{x^{2 \alpha}}{\Gamma(1+2 \alpha)} \frac{t^{2 \alpha}}{\Gamma(1+2 \alpha)}, \\
u_{2}(x, t)=\frac{x^{2 \alpha}}{\Gamma(1+2 \alpha)} \frac{t^{4 \alpha}}{\Gamma(1+4 \alpha)}, \\
u_{3}(x, t)=\frac{x^{2 \alpha}}{\Gamma(1+2 \alpha)} \frac{t^{3 \alpha}}{\Gamma(1+6 \alpha)}, \\
u_{4}(x, t)=\frac{x^{2 \alpha}}{\Gamma(1+2 \alpha)} \frac{t^{8 \alpha}}{\Gamma(1+8 \alpha)}, \\
u_{n}(x, t)=\frac{x^{2 \alpha}}{\Gamma(1+2 \alpha)} \frac{t^{2 n \alpha}}{\Gamma(1+2 n \alpha)} .
\end{gathered}
$$

Consequently, the exact solution is given by

$$
\begin{aligned}
u(x, t) & =\lim _{n \rightarrow \infty} \sum_{n=0}^{\infty} u_{n}(x, t) \\
& =\lim _{n \rightarrow \infty} \sum_{n=0}^{\infty} \frac{x^{2 \alpha}}{\Gamma(1+2 \alpha)} \frac{t^{2 n \alpha}}{\Gamma(1+2 n \alpha)} \\
& =\frac{x^{2 \alpha}}{\Gamma(1+2 \alpha)} \cosh _{\alpha}\left(x^{\alpha}\right),
\end{aligned}
$$

where

$$
\cosh _{\alpha}\left(x^{\alpha}\right)=\sum_{n=0}^{\infty} \frac{t^{2 n \alpha}}{\Gamma(1+2 n \alpha)} .
$$

The solution of (2) for $\alpha=\ln 2 / \ln 3$ is depicted in Figure 1.

\section{Conclusions}

In this work, we developed a comparison between the variational iteration method and the decomposition method within local fractional operators. The two approaches constitute efficient tools to handle the approximation solutions for differential equations on Cantor sets with local fractional derivative. We notice that the fractional variational iteration method gives the several successive approximate formulas using the iteration of the correction local fractional functional. However, the local fractional decomposition method 


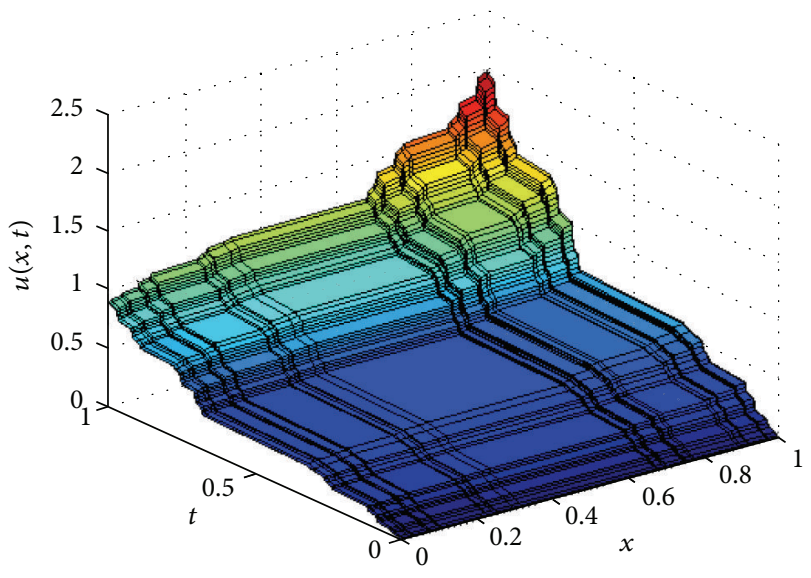

FIGURE 1: Graph of $u(x, t)$ for $\alpha=\ln 2 / \ln 3$.

provides the components of the exact solution, which is local fractional continuous function, where these components are also local fractional continuous functions. Both the variational iteration method and the decomposition method within local fractional operators provide the solution in successive components. The methods are structured to get the local fractional series solution, which is a nondifferentiable function.

\section{Conflict of Interests}

The authors declare that there is no conflict of interests regarding publication of this paper.

\section{References}

[1] A. M. Wazwaz, Partial Differential Equations: Methods and Applications, Elsevier, Balkema, The Netherlands, 2002.

[2] W. R. Schneider and W. Wyss, "Fractional diffusion and wave equations," Journal of Mathematical Physics, vol. 30, no. 1, pp. 134-144, 1989.

[3] Z. G. Zhao and C. P. Li, "Fractional difference/finite element approximations for the time-space fractional telegraph equation," Applied Mathematics and Computation, vol. 219, no. 6, pp. 2975-2988, 2012.

[4] S. Momani, Z. Odibat, and A. Alawneh, "Variational iteration method for solving the space- and time-fractional KdV equation," Numerical Methods for Partial Differential Equations, vol. 24, no. 1, pp. 262-271, 2008.

[5] N. Laskin, "Fractional Schrödinger equation," Physical Review E, vol. 66, Article ID 056108, 2002.

[6] Y. Zhou and F. Jiao, "Nonlocal Cauchy problem for fractional evolution equations," Nonlinear Analysis: Real World Applications, vol. 11, no. 5, pp. 4465-4475, 2010.

[7] S. Momani and Z. Odibat, "Analytical solution of a timefractional Navier-Stokes equation by Adomian decomposition method," Applied Mathematics and Computation, vol. 177, no. 2, pp. 488-494, 2006.

[8] V. E. Tarasov, "Fractional Heisenberg equation," Physics Letters A, vol. 372, no. 17, pp. 2984-2988, 2008.
[9] A. K. Golmankhaneh, A. K. Golmankhaneh, and D. Baleanu, "On nonlinear fractional KleinGordon equation," Signal Processing, vol. 91, no. 3, pp. 446-451, 2011.

[10] Z. B. Li, W. H. Zhu, and L. L. Huang, "Application of fractional variational iteration method to time-fractional Fisher equation," Advanced Science Letters, vol. 10, no. 1, pp. 610-614, 2012.

[11] J. Hristov, "Heat-balance integral to fractional (half-time) heat diffusion sub-model," Thermal Science, vol. 14, no. 2, pp. 291-316, 2010.

[12] D. Baleanu, K. Diethelm, E. Scalas, and J. J. Trujillo, Fractional Calculus Models and Numerical Methods, vol. 3 of Series on Complexity, Nonlinearity and Chaos, World Scientific, Boston, Mass, USA, 2012.

[13] C. Cattani, "Harmonic wavelet solution of Poisson's problem," Balkan Journal of Geometry and Its Applications, vol. 13, no. 1, pp. 27-37, 2008.

[14] C. Cattani, "Harmonic wavelets towards the solution of nonlinear PDE," Computers \& Mathematics with Applications, vol. 50, no. 8-9, pp. 1191-1210, 2005.

[15] X. J. Yang, Local Fractional Functional Analysis and Its Applications, Asian Academic, Hong Kong, China, 2011.

[16] X. J. Yang and D. Baleanu, "Fractal heat conduction problem solved by local fractional variation iteration method," Thermal Science, vol. 17, no. 2, pp. 625-628, 2013.

[17] W.-H. Su, D. Baleanu, X.-J. Yang, and H. Jafari, "Damped wave equation and dissipative wave equation in fractal strings within the local fractional variational iteration method," Fixed Point Theory and Applications, vol. 2013, article 89, 2013.

[18] X. J. Yang, D. Baleanu, and W. P. Zhong, "Approximation solutions for diffusion equation on Cantor time-space," Proceeding of the Romanian Academy A, vol. 14, no. 2, pp. 127-133, 2013.

[19] X. J. Yang, D. Baleanu, and J. A. T. Machado, "Mathematical aspects of Heisenberg uncertainty principle within local fractional Fourier analysis," Boundary Value Problems, no. 1, pp. 131146, 2013

[20] J. Klafter, S. C. Lim, and R. Metzler, Fractional Dynamics: Recent Advances, World Scientific, Singapore, 2012.

[21] J. Sabatier, O. P. Agrawal, and J. T. Machado, Advances in Fractional Calculus: Theoretical Developments and Applications in Physics and Engineering, Springer, 2007.

[22] R. Hilfer, Applications of Fractional Calculus in Physics, Word Scientific, Singapore, 2000.

[23] M. Li, S. C. Lim, and S. Chen, "Exact solution of impulse response to a class of fractional oscillators and its stability," Mathematical Problems in Engineering, vol. 2011, Article ID 657839, 9 pages, 2011.

[24] K. M. Kolwankar and A. D. Gangal, "Local fractional FokkerPlanck equation," Physical Review Letters, vol. 80, no. 2, pp. 214217, 1998.

[25] K. M. Kolwankar and A. D. Gangal, "Fractional differentiability of nowhere differentiable functions and dimensions," Chaos, vol. 6 , no. 4, pp. 505-513, 1996.

[26] A. Babakhani and V. Daftardar-Gejji, "On calculus of local fractional derivatives," Journal of Mathematical Analysis and Applications, vol. 270, no. 1, pp. 66-79, 2002.

[27] Y. Chen, Y. Yan, and K. Zhang, "On the local fractional derivative," Journal of Mathematical Analysis and Applications, vol. 362, no. 1, pp. 17-33, 2010.

[28] A. Carpinteri, B. Chiaia, and P. Cornetti, "Static-kinematic duality and the principle of virtual work in the mechanics of 
fractal media," Computer Methods in Applied Mechanics and Engineering, vol. 191, no. 1-2, pp. 3-19, 2001.

[29] A. Carpinteri, B. Chiaia, and P. Cornetti, "The elastic problem for fractal media: basic theory and finite element formulation," Computers and Structures, vol. 82, no. 6, pp. 499-508, 2004.

[30] A. Carpinteri and P. Cornetti, "A fractional calculus approach to the description of stress and strain localization in fractal media," Chaos, Solitons \& Fractals, vol. 13, no. 1, pp. 85-94, 2002.

[31] F. Ben Adda and J. Cresson, "About non-differentiable functions," Journal of Mathematical Analysis and Applications, vol. 263, no. 2, pp. 721-737, 2001.

[32] W. Chen, "Time-space fabric underlying anomalous diffusion," Chaos, Solitons \& Fractals, vol. 28, no. 4, pp. 923-929, 2006.

[33] W. Chen, H. G. Sun, X. D. Zhang, and D. Korošak, "Anomalous diffusion modeling by fractal and fractional derivatives," Computers \& Mathematics with Applications, vol. 59, no. 5, pp. 1754-1758, 2010.

[34] G. Jumarie, "Modified Riemann-Liouville derivative and fractional Taylor series of nondifferentiable functions further results," Computers \& Mathematics with Applications, vol. 51, no. 9-10, pp. 1367-1376, 2006.

[35] X. J. Yang, Advanced Local Fractional Calculus and Its Applications, World Science, New York, NY, USA, 2012.

[36] A.-M. Yang, X.-J. Yang, and Z.-B. Li, "Local fractional series expansion method for solving wave and diffusion equations on Cantor sets," Abstract and Applied Analysis, vol. 2013, Article ID 351057, 5 pages, 2013. 


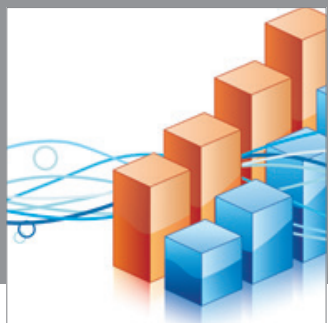

Advances in

Operations Research

mansans

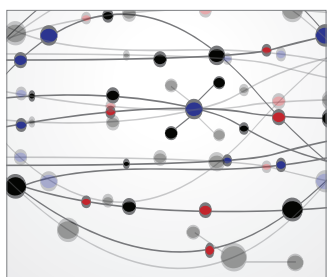

The Scientific World Journal
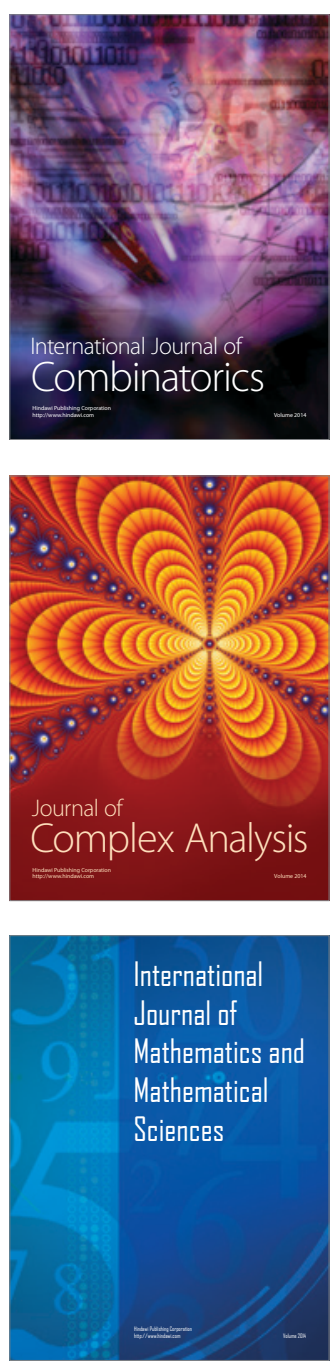
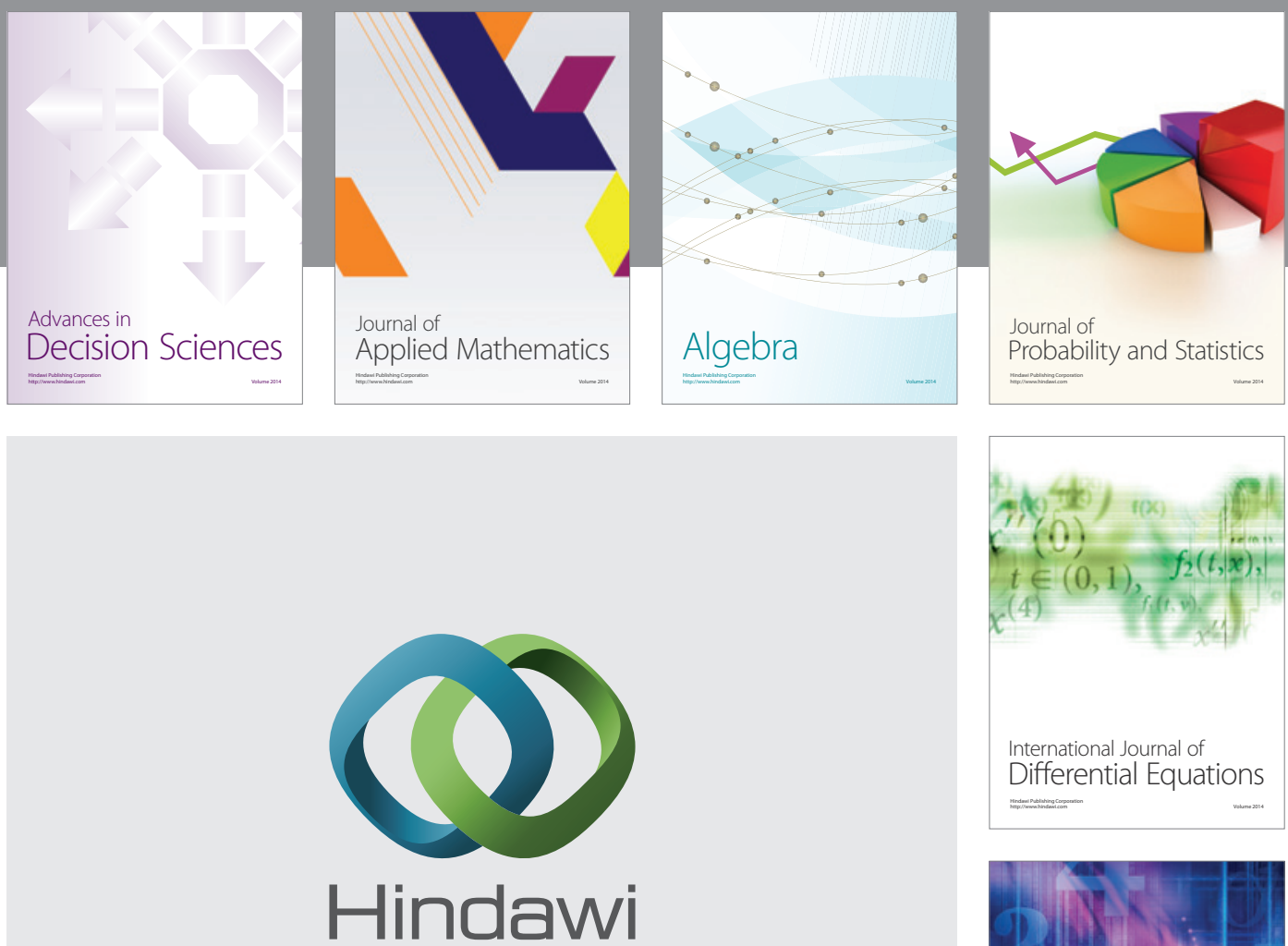

Submit your manuscripts at http://www.hindawi.com
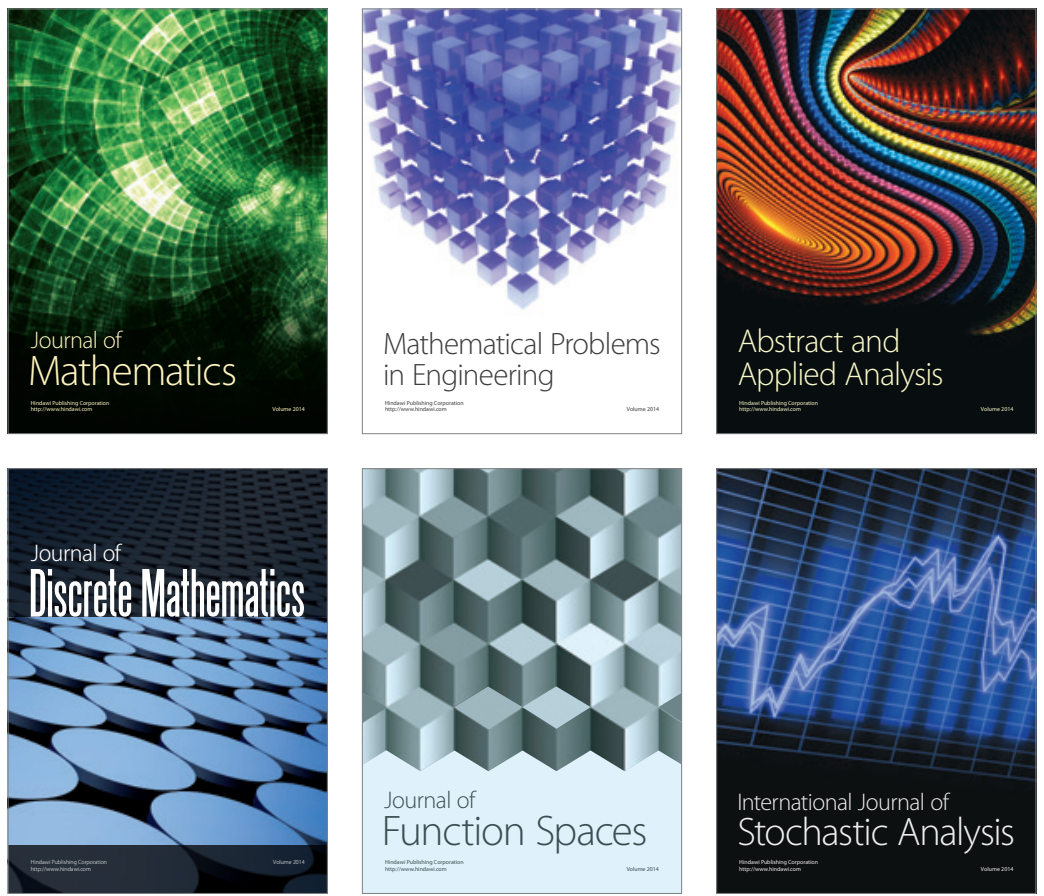

Journal of

Function Spaces

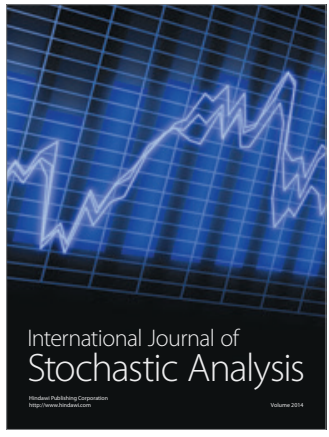

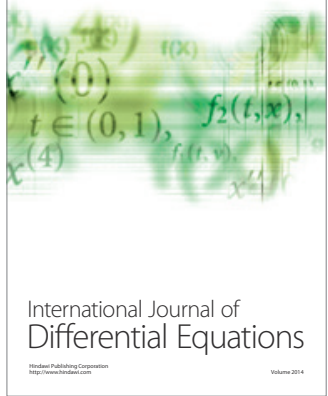
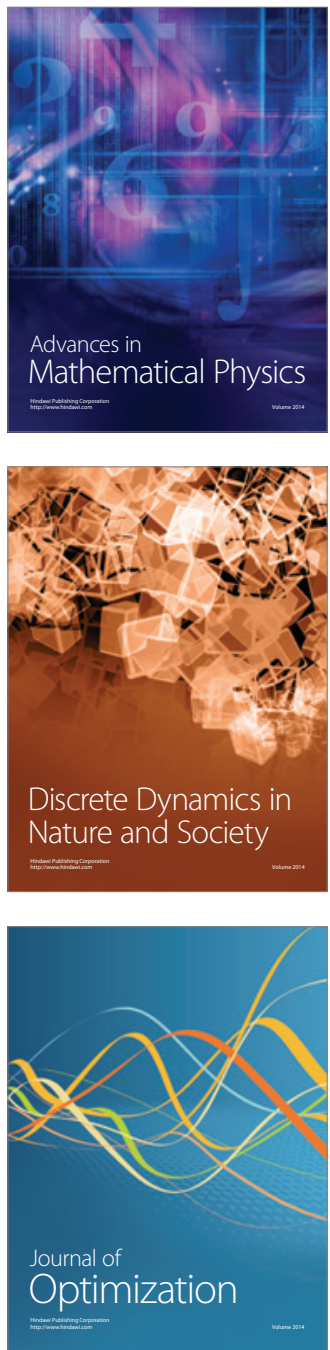